\title{
On The Spectrum of Schrödinger Operators with a Random Potential
}

\author{
Werner Kirsch ${ }^{1}$ and Fabio Martinelli ${ }^{2}$ \\ 1 Institut für Mathematik, Ruhr-Universität Bochum, D-4630 Federal Republic of Germany \\ 2 Istituto di Fisica G. N. F. M., Università di Roma, Roma, Italy
}

\begin{abstract}
We investigate the spectrum of Schrödinger operators $H_{\omega}$ of the type: $H_{\omega}=-\Delta+\sum q_{i}(\omega) f\left(x-x_{i}+\xi_{i}(\omega)\right)\left(q_{i}(\omega)\right.$ and $\xi_{i}(\omega)$ independent identically distributed random variables, $\left.i \in \mathbb{Z}^{d}\right)$. We establish a strong connection between the spectrum of $H_{\omega}$ and the spectra of deterministic periodic Schrödinger operators. From this we derive a condition for the existence of "forbidden zones" in the spectrum of $H_{\omega}$. For random one- and three-dimensional Kronig-Penney potentials the spectrum is given explicitly.
\end{abstract}

\section{Introduction}

In this paper we study the spectra of random Schrödinger operators $H_{\omega}$ of the form:

$$
H_{\omega}=-\Delta+\sum q_{i}(\omega) f\left(x-x_{i}+\xi_{i}(\omega)\right),
$$

where $\left\{x_{i}\right\}_{i \in \mathbb{Z}^{d}}$ is a Bravais Lattice and $\left\{q_{i}\right\}_{i \in \mathbb{Z}^{d}}$ and $\left\{\xi_{i}\right\}_{i \in \mathbb{Z}^{d}}$ are independent, identically distributed random variables. Physically speaking $H_{\omega}$ corresponds to a random "charge"-configuration $\left\{q_{i}(\omega)\right\}$, each $q_{i}(\omega)$ being located at the random position $x_{i}-\xi_{i}(\omega)$ and producing a potential $q_{i}(\omega) f\left(x-x_{i}+\xi_{i}(\omega)\right)$. Thus $H_{\omega}$ can be used as the Hamiltonian of a model for a "mixed" crystal with centers of strength $q_{i}(\omega)$ at perturbed lattice positions $x_{i}-\xi_{i}(\omega)$ or of a model of a liquid.

Models of this kind were considered by many authors, see for example: Halperin [10], Frisch and Lloyd [7], Luttinger [15], Borland [4], Lieb and Mattis [14] and references therein. Random operators of a more or less different kind are studied e.g. in Pastur [18] and [19], Kunz and Souillard [13], Fukushima, Nagai and Nakao [8], Nakao [17] and references given there.

In [11] the present authors showed that the spectrum of a wide class of random operators, containing the $H_{\omega}$ given above, is a nonrandom set $\Sigma$. In the present paper we determine the spectrum of the above operator more precisely.

In the first section we give conditions under which the operator $H_{\omega}$ is well defined and moreover essentially self-adjoint on $C_{0}^{\infty}\left(\mathbb{R}^{d}\right)$, the infinitely differentiable 
functions with compact support. This turns out to be the case if the moments of $\left\{q_{i}\right\}$ up to a sufficiently high order are finite and the function $f$ is not too singular.

In Sect. 2 we investigate the relation between the spectrum of $H_{\omega}$ and the spectra of well ordered "charge" configurations, i.e. of Schrödinger operators of the form

$$
H_{\lambda, u}=-\Delta+\sum \lambda_{i} f\left(x-x_{i}+u_{i}\right)
$$

where $\lambda_{i}$ as well as $u_{i}$ are periodic and nonrandom. We get that the set $\Sigma$, the spectrum of $H_{\omega}$, is completely determined by the spectra of operators of the form $H_{\lambda, u}$. Thus $\Sigma$ has a band structure, in the sense that $\Sigma=\cup\left[a_{i}, b_{i}\right]$ but the intervals $\left[a_{i}, b_{i}\right]$ may overlap.

In Sect. 3 for $\xi_{i} \equiv 0$ we give a sufficient condition for the existence of forbidden zones ("gaps") in $\Sigma$ (Theorem 5). Under a very mild condition (Assumption A) on $f$ and $\left\{q_{i}\right\}$ it is shown that $(\alpha, \beta)$ is a gap for $H_{\omega}$ if it is a gap for all "pure" Hamiltonians $H_{\lambda}=-\Delta+\lambda \Sigma f\left(x-x_{i}\right)$, where $\lambda$ runs through the (connected) component of supp $p_{q_{0}}^{\prime}$ the support of the probability distribution of $q_{0}(\omega)$. Theorem 5 can be looked upon as a generalisation of a famous conjecture by Saxon and Hutner [21].

These authors conjectured that a common gap for two pure solids is also a gap for an alloy of these solids, at least in a one-dimensional model with point interactions. In the latter case the conjecture was proved by Luttinger [15], but it was shown to be wrong for other potentials (see e.g.: Lieb and Mattis [14], Halperin [10]).

In Sect. 4 we study three examples. First we choose $f$ to be a square-well potential in one dimension without overlapping of the wells. For this the existence of infinitely many gaps is shown. The second example is a random point interaction in one dimension, a generalisation of the model considered by Luttinger [15], the nonrandom version of which goes back to Kronig and Penney [12]. Specifically we give the spectrum of $H_{\omega}$, as in the nonrandom case. This result contains Luttinger's result mentioned above. Our last example shows the existence of a gap for a random point interaction in three dimensions.

\section{Section 1}

Let $\left\{q_{i}(\omega)\right\}_{i \in \mathbb{Z}^{a}}$ be independent, identically distributed random variables on a probability space $(\Omega, \mathscr{F}, P)$. Let $\left\{x_{i}\right\}_{i \in \mathbb{Z}^{d}}$ be a Bravais Lattice, i.e. $i \rightarrow x_{i}$ is a representation of the group $\mathbb{Z}^{d}$ into $\mathbb{R}^{d}$ such that the $\left\{x_{i}\right\}_{i \in \mathbb{Z}^{d}}$ span the space $\mathbb{R}^{d}$. By introducing a new norm on $\mathbb{R}^{d}$, if necessary, we can assume the lattice $\left\{x_{i}\right\}_{i \in \mathbb{Z}^{d}}$ to be $\mathbb{Z}^{d}$. Furthermore let $f$ be a real $L_{\text {loc }}^{p}\left(\mathbb{R}^{d}\right)$-function, for some $p \geqq d / 2$ for $d \geqq 4$, and $p=2$ for $d \leqq 3$, such that:

$$
\sum_{\substack{i \in \mathbb{Z}^{d} \\|i| \geqq k_{0}}} \sup _{x \in C_{0}+x_{l}}|f(x)|<\infty
$$

for some constant $K_{0}$; where $C_{0}$ is the unit cube around the point $x_{0}=0$.

Then we define the potential $V_{\omega}(x)$ by:

$$
V_{\omega}(x)=\sum_{i \in \mathbb{Z}^{d}} q_{i}(\omega) f\left(x-x_{i}\right)
$$


Clearly we have to put some restrictions on the random variables $\left\{q_{i}\right\}_{i \in \mathbb{Z}^{d}}$ in order that $V_{\omega}(x)$ is well defined.

Lemma 1. If the first two moments of $q_{0}(\omega)$ are finite, i.e. $E\left|q_{0}(\omega)\right|<+\infty, E\left|q_{0}(\omega)\right|^{2}<+\infty$, where $E$ denotes the expectation with respect to the probability measure $P$, then $\sum_{i \in \mathbb{Z}^{d}}\left|q_{i}(\omega)\right|\left|f\left(x-x_{i}\right)\right|$ is a locally $L^{2}\left(\mathbb{R}^{d}\right)$-function with probability one.

The proof is omitted since it is standard. From the above Lemma we get that $V_{\omega}(x)$ is a well-defined function locally square integrable for almost all $\omega \in \Omega$. Hence the operator: $H_{\omega}=-\Delta+V_{\omega}$ on $L^{2}\left(R^{d}\right)$, where $\Delta$ denotes the Laplacian, is a densely defined (e.g. on $C_{0}^{\infty}\left(\mathbb{R}^{d}\right)$ ) symmetric operator on $L^{2}\left(\mathbb{R}^{d}\right)$ and by $V$. Neumann's theorem.(see e.g. Reed-Simon II [20]) has selfadjoint extensions.

Actually with mild additional assumptions on $f$ and on $\left\{q_{i}\right\}_{i \in \mathbb{Z}^{d}}$ we can prove the following result:

Theorem 1. Let the real function $f(x)$ on $\mathbb{R}^{d}$ be as before (i.e. $f$ satisfies assumption (1) and $f \in L^{p}\left(\mathbb{R}^{d}\right)$ for some $p>2$ for $d \leqq 3$ and $p>d / 2$ for $d>3$ ) and assume that $E\left|q_{0}\right|^{k}<$ $+\infty$ with $k>p d / 2(p-2)$ for $d \leqq 3$ and $k>d p /(2 p-d)$ for $d>3$. Then the Hamiltonian $-\Delta+\sum_{i \in \mathbb{Z}^{d}} q_{i}(\omega) f\left(x-x_{i}\right)$ on $L^{2}\left(\mathbb{R}^{d}\right)$ is essentially selfadjoint on $C_{0}^{\infty}\left(\mathbb{R}^{d}\right)$ with probability one.

Proof. For simplicity we assume the constant $k_{0}$ appearing in (1) equal to 1 . The idea of the proof is to show that with probability one it is possible to split $V_{\omega}$ into: $V_{\omega}=V_{\omega}^{(1)}+V_{\omega}^{(2)}$, in such a way that $V_{\omega}^{(1)}(x)>-c(\omega) x^{2}$ for some positive constant $c(\omega)$ and $V_{\omega}^{(2)} \in L_{\text {un,loc }}^{q}\left(\mathbb{R}^{d}\right)$, i.e. $\int_{c_{0}+y}\left|V_{\omega}^{(2)}(x)\right|^{q} d x<c_{1}$ for any $y \in \mathbb{R}^{d}$ and a constant $c_{1}=c_{1}(\omega)$, independent of $y$, for some $q>d / 2$ if $d>3, q=2$ if $d \leqq 3$. Then the essential selfadjointness follows from the Faris-Lavine theorem (see e.g. Reed-Simon II [20]) combined with Theorem XIII 96 of Reed-Simon IV [20].

We will treat only the case $d \geqq 3$; the other cases, i.e. $d=2$ or $d=1$, can be handled exactly in the same way.

By definition, $V_{\omega}(x)$ can be written as:

$$
\begin{aligned}
V_{\omega}(x) & =\sum_{j \neq i(x)} q_{j}(\omega) f\left(x-x_{j}\right)+q_{i(x)} f\left(x-x_{i(x)}\right) \\
& \equiv \bar{V}_{\omega}(x)+q_{i(x)} f\left(x-x_{i(x)}\right),
\end{aligned}
$$

where $i(x)$ is such that $x \in C_{0}+x_{i(x)} . \bar{V}_{\omega}(x)$ is the non-singular part of $V_{\omega}(x)$ and by assumption (1) is finite for almost every $\omega \in \Omega$.

We will show that:

From (4) we can conclude that:

$$
\begin{aligned}
& P\left(\sum_{j \neq i}\left|q_{j}(\omega)\right| \sup _{x \in C_{0}+x_{i}}\left|f\left(x-x_{j}\right)\right|>\inf _{x \in C_{0}+x_{i}}|x|^{2}\right. \\
& \text { for infinitely many } \left.x_{i}\right)=0 .
\end{aligned}
$$

$$
\sup _{x \in C_{0}+x_{i}}\left|\bar{V}_{\omega}(x)\right|>\inf _{x \in C_{0}+x_{i}}|x|^{2} \text { only for finitely many } x_{i}
$$


almost surely; hence, since $\bar{V}_{\omega}(x)$ is finite almost surely we can find with probability one a positive constant $C(\omega)$ such that

$$
\bar{V}_{\omega}(x)>-C(\omega)|x|^{2} .
$$

By the Borel-Cantelli Lemma in order to prove (4) it suffices to show for a suitable chosen $M$ :

$$
\sum_{\substack{i \in \mathbb{Z}^{d} \\|i|>M}} P\left(\sum_{j \neq i}\left|q_{j}\right| \sup _{x \in C_{0}+x_{i}}\left|f\left(x-x_{j}\right)\right|>\inf _{x \in C_{0}+x i}|x|^{2}\right)<+\infty ;
$$

by the Chebyshev inequality we have:

$$
\begin{gathered}
P\left(\sum_{j \neq i}\left|q_{j}\right| \sup _{x \in C_{0}+x_{i}}\left|f\left(x-x_{j}\right)\right|>\inf _{x \in C_{0}+x_{i}}|x|^{2}\right) \\
\leqq \frac{E\left(\sum_{j \neq i}\left|q_{j}\right| \sup _{x \in C_{0}+x_{i}}\left|f\left(x-x_{j}\right)\right|\right)^{k}}{\left(\inf _{x \in C_{0}+x_{i}}|x|^{2}\right)^{k}},
\end{gathered}
$$

where $k$ is as in the statement of the theorem.

The right hand side of (7) can be bounded from above by

$$
E\left|q_{0}\right|^{k} D^{k}\left|x_{i}^{\prime}\right|^{-2 k}
$$

where $D=\sum_{j \neq 0} \sup _{x \in C_{0}}\left|f\left(x-x_{j}\right)\right|<+\infty$ by assumption (1), and $x_{i}^{\prime 2}$ means $\inf _{x \in C_{0}+x^{i}}|x|^{2}$.

Inserting estimate (8) in (6) we get:

$$
\begin{gathered}
\sum_{\substack{i \in \mathbb{Z}^{d} \\
|i|>M}} P\left(\sum_{j \neq i}\left|q_{j}\right| \sup _{x \in C_{0}+x_{i}}\left|f\left(x-x_{i}\right)\right|>\inf _{x \in C_{0}+x_{i}}|x|^{2}\right) \\
\leqq E\left|q_{0}\right|^{k} D^{k} \sum_{\substack{i \in \mathbb{Z}^{d} \\
|i|>M}}\left|x_{i}^{\prime}\right|^{-2 k}<+\infty
\end{gathered}
$$

since $k>d p /(2 p-d)>d / 2$ in the case $d>3$, and $k>d p / 2(p-2)>d / 2$ in the case $d=3$.

We now consider the singular part of $V_{\omega}(x): \tilde{V}_{\omega}(x) \equiv q_{i(x)} f\left(x-x_{i(x)}\right)$, where as before $i(x)$ is such that $x \in C_{0}+x_{i(x)} . \tilde{V}_{\omega}(x)$ in turn can be decomposed as follows:

$$
\begin{aligned}
\tilde{V}_{\omega}(x)= & q_{i(x)}^{(\omega)} f\left(x-x_{i(x)}\right) \chi\left(q_{i(x)}^{(\omega)} f\left(x-x_{i(x)}\right) \geqq-x^{2}\right) \\
& +q_{i(x)}^{(\omega)} f\left(x-x_{i(x)}\right) \chi\left(q_{i(x)}^{(\omega)} f\left(x-x_{i(x)}\right)<-x^{2}\right) .
\end{aligned}
$$

Here $\chi(\cdot)$ denotes the characteristic function on $\mathbb{R}^{d}$.

The proof of the theorem is now complete if we are able to prove that $q_{i(x)}^{(\omega)} f\left(x-x_{i(x)}\right) \chi\left(q_{i(x)}^{(\omega)} f\left(x-x_{i(x)}\right)<-x^{2}\right)$ belongs to $L_{\mathrm{un}, 1 \mathrm{loc}}^{q}\left(\mathbb{R}^{d}\right)$ almost surely, for some $q>d / 2$ for $d>3, q=2, d=3$. For this it is sufficient to show:

$$
\int_{c_{0}+x_{i}}\left|q_{i}(\omega)\right|^{q}\left|f\left(x-x_{i}\right)\right|^{q} \chi\left(\left|q_{i}\right|\left|f\left(x-x_{i}\right)\right|>x^{2}\right) d x<M_{1}(\omega)
$$

for some $q>d / 2$ for $d>3, q=2$ for $d=3$ and some constant $M_{1}(\omega)$ independent of $i \in \mathbb{Z}^{d}$ with probability one. Since $f \in L_{\mathrm{loc}}^{p}\left(\mathbb{R}^{d}\right)$ with $p>d / 2$ for $d>3$ and $p>2$ for 
$d=3$, we can choose $q$ in such a way that $\max (2, d / 2)<q<p$ for $d \geqq 3$. As before it is enough to check that:

$$
P\left(\int_{c_{0}+x_{i}}\left|q_{i}\right|^{q}\left|f\left(x-x_{i}\right)\right|^{q} \chi\left(\left|q_{i}\right|\left|f\left(x-x_{i}\right)\right|>x^{2}\right) d x>1\right.
$$

for infinitely many $\left.i \in \mathbb{Z}^{d}\right)=0$.

In fact if (12) is true then $\int_{C_{0}+x_{i}}\left|q_{i}\right|^{q}\left|f\left(x-x_{i}\right)\right|^{q} \chi\left(\left|q_{i}\right|\left|f\left(x-x_{i}\right)\right|>x^{2}\right) d x>1$ only for finitely many $i \in Z^{d}$, and since $f(x) \in L_{\text {loc }}^{p}\left(\mathbb{R}^{d}\right)$ for $d / 2<q<p$ in the case $d>3$, and $f \in L_{\text {loc }}^{2}\left(\mathbb{R}^{d}\right)$ for $d=3$, it is always possible to find a constant $M_{1}(\omega)$ such that (11) is satisfied for any $i \in \mathbb{Z}^{d}$.

Again, by the Borel-Cantelli lemma, the problem is reduced to give an estimate of:

$$
P\left(\int_{C_{0}+x_{i}}\left|q_{i}\right|^{q}\left|f\left(x-x_{i}\right)\right|^{q} \chi\left(\left|q_{i}\right|\left|f\left(x-x_{i}\right)\right|>x^{2}\right) d x>1\right)
$$

such that the sum:

$$
\sum_{\substack{i \in \mathbb{Z} \\|i|>M_{0}}} P\left(\int_{C_{0}+x_{i}}\left|q_{i}\right|^{q}\left|f\left(x-x_{i}\right)\right|^{q} \chi\left(\left|q_{i}\right| \mid f\left(x-x_{i}\right)>x^{2}\right) \mid d x>1\right)
$$

is finite. By the Hölder inequality the integral appearing in formula (13) can be estimated from above by:

$$
\begin{aligned}
& \left|q_{i}\right|^{q}\left[\int_{C_{0}+x_{2}}\left|f\left(x-x_{i}\right)\right|^{p} d x\right]^{q / p}\left[\int_{C_{0}+x_{2}} \chi\left(\left|q_{i}\right|\left|f\left(x-x_{i}\right)\right|>x^{2}\right) d x\right]^{1-q / p} \\
& \quad \leqq\left|q_{i}\right|^{p}\left[\int_{C_{0}+x_{i}}\left|f\left(x-x_{i}\right)\right|^{p} d x\right]^{q / p} b_{1} x_{i}^{\prime}-2 p(1-q / p)
\end{aligned}
$$

for some constant $b_{1}>0$, where we have used the fact that $f \in L_{w}^{p}\left(C_{0}\right)($ see e.g. ReedSimon II page 30, [20]). Using now the Chebyshev inequality and estimate (15) we obtain:

$$
\begin{aligned}
& P\left(\int_{C_{0}+x_{i}}\left|q_{i}\right|^{q}\left|f\left(x-x_{i}\right)\right|^{q} \chi\left(\left|q_{i}\right|\left|f\left(x-x_{i}\right)\right|>x^{2}\right) d x>1\right) \\
& \quad \leqq b_{2} E\left|q_{0}\right|^{k}\left|x_{i}^{\prime}\right|^{-2 k(1-q / p)}
\end{aligned}
$$

for some constant $b_{2}>0$, where $K$ is as in the statement of the theorem.

Inserting (16) in (14) we get that:

$$
\begin{gathered}
\sum_{\substack{i \in \mathbb{Z}^{d} \\
|i|>M_{0}}} P\left(\int_{C_{0}+x_{i}}\left|q_{i}\right|^{q}\left|f\left(x-x_{i}\right)\right|^{q} \chi\left(\left|q_{i}\right|\left|f\left(x-x_{i}\right)\right|>x^{2}\right) d x>1\right) \\
\leqq \sum_{\substack{i \in \mathbb{Z}^{d} \\
|i|>M_{0}}} b_{2} E\left|q_{0}\right|^{k}\left|x_{i}^{\prime}\right|^{-2 k(1-q / p)}
\end{gathered}
$$

is finite if $2 k(1-q / p)>d$, i.e. if $q<p-d p / 2 k$. Since $k>d p /(2 p-d)$ for $d>3$, $k>d p / 2(p-2)$ for $d=3, p-d p / 2 k>d / 2$ for $d>3$ and $p-d p / 2 k>2$ for $d=3$, we can always find a $p$ such that: $d / 2<p<p-d p / 2 k$ for $d>3$ and $2<p<p-d p / 2 k$ for $d=3$. The convergence of (14) is thus assured. 
Remark. The results of this section, namely Lemma 1 and Theorem 1 can be extended to the following case:

$$
V_{\omega}(x)=\sum q_{i}(\omega) f\left(x-x_{i}+\xi_{i}(\omega)\right),
$$

where $\left\{\xi_{i}\right\}_{i \in \mathbb{Z}^{d}}$ are new independent, identically distributed random variables satisfying $\left|\xi_{i}(\omega)\right|<N_{0}$ for any $\omega$.

\section{Section 2}

In this section we investigate the spectra of Schrödinger operators of the form $H_{0}=$ $-\Delta+V_{\omega}$ with $V_{\omega}$ as in (17) with the stronger assumption that the $\left\{q_{i}(\omega)\right\}_{i \in \mathbb{Z}^{d}}$ are independent identical distributed random variables. The following theorem was proved in Kirsch-Martinelli [11]:

For almost each $\omega \in \Omega$, let $H_{\omega}$ be a selfadjoint operator on a separable Hilbert space $H$, such that for each $z \in \mathbb{R}$ the function $\omega \rightarrow\left(z-H_{\omega}\right)^{-1}$ is weakly measurable. Furthermore assume that there exist measure preserving transformations $\left\{T_{i}\right\}_{i \in I}$ ( $I$ an arbitrary index set), which are ergodic in the sense that every $A \in \mathscr{F}$ which is invariant under all $\left\{T_{i}\right\}_{i \in \mathbb{Z}^{d}}$ has either probability one or probability zero. Suppose furthermore that there are unitary operators. $\left\{U_{i}\right\}_{i \in I}$ on $H$ such that

Then we have

$$
H_{T_{\iota} \omega}=U_{i} H_{\omega} U_{i}^{*}
$$

Theorem 2 (Theorem 1 and 2 of [11]).

1) The spectrum $\sigma\left(H_{\omega}\right)$ of $H_{\omega}$ is a nonrandom set.

2) The pure point part, the singular continuous part and the absolutely continuous part of $\sigma\left(H_{\omega}\right)$ are nonrandom sets.

This theorem can be applied to our situation, since the $\left\{q_{i}\right\}_{i \in \mathbb{Z}^{d}}$ (as well as the $\left.\left\{\xi_{i}\right\}_{i \in \mathbb{Z}^{d}}\right)$ as independent identically distributed random variables form a stationary, metrically transitive random field, i.e. there are measure preserving, ergodic transformations $\left\{T_{i}\right\}_{i \in \mathbb{Z}^{d}}$ such that

$$
q_{j}\left(T_{i} \omega\right)=q_{j-i}(\omega)
$$

Hence with: $U_{i} f(x):=f\left(x-x_{i}\right)$ one has immediately:

$$
H_{T_{\imath} \omega}=-\Delta+V_{T_{\imath} \omega}=-\Delta+U_{i} V_{\omega} U_{i}^{*}=U_{i}\left(-\Delta+V_{\omega}\right) U_{i}^{*}=U_{i} H_{\omega} U_{i}^{*} .
$$

The measurability of $\left(z-H_{\omega}\right)^{-1}$ can be obtained by Corollary 3 in [11]. There it was proved that $\left(z-\left(-\Delta+V_{\omega}\right)\right)^{-1}$ is weakly measurable if $V$ as a function of $\omega$ and $x$ is measurable and $-\Delta+V_{\omega}$ is essentially selfadjoint on $C_{0}^{\infty}\left(\mathbb{R}^{d}\right)$ almost surely. By Theorem 1 we know that in our case $H_{\omega}=-\Delta+V_{\omega}$ is essentially selfadjoint on $C_{0}^{\infty}\left(\mathbb{R}^{d}\right)$, hence Theorem 2 holds for this operators. Moreover an application of Corollary 1 in [11] shows that the discrete part of the spectrum of $H_{\omega}$ is almost surely empty.

Applications of Theorem 2 to more general random operators were discussed in [11]. In our special case however it is possible to investigate the spectrum of $H_{\omega}$ more precisely. In doing this the following definition is convenient.

Definition. We call a real function $W$ on $\mathbb{R}^{d}$ an admissible potential for the operator 
$H_{\omega}:=-\Delta+V_{\omega}\left(V_{\omega}\right.$ as defined in (17)) if $W(x)=\Sigma c_{i} f\left(x-x_{i}+u_{i}\right)$ with nonrandom $c_{i} \in \mathbb{R}, u_{i} \in \mathbb{R}^{d}$ and the following conditions are satisfied:

1) $c_{i} \in \operatorname{supp} P_{q_{i}}$, where $P_{q_{i}}$ is the (common) distribution of $q_{i}$.

2) $u_{i} \in \operatorname{supp} P_{\xi_{1}}$, where $P_{\xi_{1}}$ is the (common) distribution of $\xi_{i}$.

3) $W$ is locally square integrable.

4) $-\Delta+W$ is essentially selfadjoint on $C_{0}^{\infty}\left(\mathbb{R}^{d}\right)$.

5) $\sum_{|i| \geqq M} c_{i} \sup _{x \in C_{0}}\left|f\left(x-x_{i}+u_{i}\right)\right|<\infty$ for $M$ large enough.

Remark. For almost every $\omega \in \Omega V_{\omega}$ is an admissible potential; conditions 3) and 5) follow from Lemma 1, condition 4) from Theorem 1.

Now we prove the following theorem which allows an investigation of the spectrum of $H_{\omega}$ by means of admissible potentials.

Theorem 3. If $W$ is an admissible potential for $H_{\omega}$ the spectrum $\sigma(-\Delta+W)$ of $-\Delta$ $+W$ is contained in the set $\Sigma$, which is the spectrum of $H_{\omega}$ almost surely. Thus $\Sigma=\bigcup_{W \in A} \sigma(-\Delta+W)$

where the union is taken over the class $A$ of all admissible potentials.

Proof. Let $A:=\left\{\omega \in \Omega ; \underset{N \in \mathbb{N}}{\forall} \underset{k \in \mathbb{N}}{\forall} \underset{x_{0}(N, k, \omega) \in \mathbb{Z}^{d}}{\exists}\right.$

$$
\left.\int_{B_{N}}\left|W(x)-V\left(x+x_{0}(N, k, \omega), \omega\right)\right|^{2} d x<1 / k\right\} .
$$

where $B_{N}:=\{x ;|x| \leqq N\}$.

In Lemma 2 we will show that $P(A)=1$. Take now $\lambda \in \sigma(-\Delta+w)$, then by the Weyl criterion (see e.g. Weidmann [22] Theorem 7.22) there exists a sequence $\left\{\varphi_{k}\right\}_{k \in \mathbb{N}}$ in $C_{0}^{\infty}\left(\mathbb{R}^{d}\right)$ such that: $\left\|\varphi_{k}\right\|=1\left(\|\|\right.$ denotes the $L^{2}$-norm $)$ and $\|(-\Delta$ $+W) \varphi_{k}-\lambda \varphi_{k} \|<1 / k$. Choose $N_{k}$ large enough in such a way that $\operatorname{supp} \varphi_{k} \subset B_{N_{k}}$ and take $\omega \in A \cap \Omega_{0}\left(\Omega_{0}=\left\{\omega \in \Omega ; \sigma\left(H_{\omega}\right)=\Sigma\right\}\right)$. By Theorem 2 and Lemma 2 we have $P\left(A \cap \Omega_{0}\right)=1$. Choose $x_{0}\left(N_{k}, k^{2}\left\|\varphi_{k}\right\|_{\infty}^{2}, \omega\right)$, where $\left\|\varphi_{k}\right\|_{\infty}=\sup _{x \in \mathbb{R}^{d}}\left|\varphi_{k}(x)\right|$; by the definition of $A$ we have:

$$
\int_{B_{N}}\left|W(x)-V_{\omega}\left(x+x_{0}\left(N_{k}, k^{2}\left\|\varphi_{k}\right\|_{\infty}^{2}, \omega\right)\right)\right|^{2} d x<\frac{1}{k^{2}\left\|\varphi_{k}\right\|_{\infty}^{2}} .
$$

If we define the new sequence of $C_{0}^{\infty}$-functions:

$$
\psi_{k}(x):=\varphi_{k}\left(x-x_{0}\left(N_{k}, K^{2}\left\|\varphi_{k}\right\|_{\infty}^{2}, \omega\right)\right)
$$

then: $\left\|\left(-\Delta+V_{\omega}\right) \psi_{k}-\lambda \psi_{k}\right\|$

$$
\begin{aligned}
& =\left\|\left(-\Delta+V_{\omega}\left(x+x_{0}\left(N_{k}, k^{2}\left\|\varphi_{k}\right\|_{\infty}^{2}, \omega\right)\right)\right) \varphi_{k}-\lambda \varphi_{k}\right\| \\
& \leqq\left\|(-\Delta+W) \varphi_{k}-\lambda \varphi_{k}\right\|+\left\|\left\{W(x)-V_{\omega}\left(x+x_{0}\left(N_{k}, k^{2}\left\|\varphi_{k}\right\|_{\infty}^{2}, \omega\right)\right)\right\} \varphi_{k}\right\| \\
& \leqq \frac{1}{k}+\frac{1}{k}=2 k .
\end{aligned}
$$


Hence, again by the Weyl criterion, $\lambda \in \sigma\left(-\Delta+V_{\omega}\right)=\Sigma$ since $\omega$ belongs by assumption also to $\Omega_{0}$.

It remains only to show the following lemma:

Lemma 2. Let $W$ be an admissible potential and define

$$
\begin{aligned}
A=\{ & \left\{\omega \in \Omega ; \underset{N \in \mathbb{N}}{\forall} \underset{k \in \mathbb{N}}{\forall} \exists x_{0}(N, k, \omega) \in \mathbb{Z}^{d}\right. \\
& \left.\int_{B_{N}}\left|W(x)-V_{\omega}\left(x+x_{0}(N, k, \omega)\right)\right|^{2} d x<\frac{1}{k}\right\} .
\end{aligned}
$$

Then $P(A)=1$.

Proof. Let $A_{N, k}=\left\{\omega \in \Omega ; \exists x_{0}(\omega) \int_{B_{N}}\left|W(x)-V_{\omega}\left(x+x_{0}(\omega)\right)\right|^{2} d x<\frac{1}{k}\right\}$. Since $A_{N+1, k} \subset A_{N, k}$, if we define $A_{k}=\bigcap_{N=1}^{\infty} A_{N, k}$, then $P\left(A_{k}\right)=\lim _{N \rightarrow \infty} P\left(A_{N, k}\right)$. By definition $A=\bigcap_{k=1}^{\infty} A_{k}$, and since $A_{k+1} \subset A_{k}$ we get $P(A)=\lim _{k \rightarrow \infty} P\left(A_{k}\right)$. Thus in order to show that $P(A)=1$ it suffices to show that $P\left(A_{N . k}\right)=1$ for any $N, k \in \mathbb{N}$. Clearly $A_{N, k}$ is invariant under the ergodic shift $\left\{T_{i}\right\}_{i \in \mathbb{Z}^{d}}$ on $\Omega$, so that $P\left(A_{\mathbb{N}, k}\right)$ is either equal to one or to zero. So it is enough to show that $P\left(A_{N, k}\right)>0$, or $P\left(C_{N, k}\right)>0$, where $C_{N, k} \subset A_{N, k}$ is the set $\left\{\omega \in \Omega ; \int_{B_{N}}|W(x)-V(x, \omega)|^{2} d x<1 / k\right\}$.

Let $M_{1}$ be a positive constant large enough such that:

$$
\int_{B_{N}}\left|\sum_{|i|>M_{1}} c_{i} f\left(x-x_{i}+u_{i}\right)\right|^{2} d x<1 / 4 k
$$

This constant $M_{1}$ always exists since:

$$
\begin{aligned}
& \int_{B_{N}}\left|\sum_{|i|>M} c_{i} f\left(x-x_{i}+u_{i}\right)\right|^{2} d x \\
& \quad \leqq \sum_{|j|=1}^{k(N)} \int_{C_{0}+x_{j}}\left|\sum_{|i|>M_{1}} c_{i} f\left(x-x_{i}+u_{i}\right)\right|^{2} d x \\
& \quad=\sum_{|j|=1}^{k(N)} \int_{C_{0}}\left|\sum_{|i|>M_{1}} c_{i} f\left(x-x_{j}-x_{i}+u_{i}\right)\right|^{2} d x \\
& \leqq \sum_{|j|=1}^{k(N)}\left(\sum_{|i|>M_{1}}\left|c_{i}\right| \sup _{x \in C_{0}}\left|f\left(x+x_{j}-x_{i}+u_{i}\right)\right|\right)^{2}
\end{aligned}
$$

and this last term can be made arbitrarily small because of point (5) of the definition of admissible potential.

Next, consider the set

$$
D=\left\{\omega \in \Omega ; \exists M \int_{B_{N}}\left(\sum_{|i|>M}\left|q_{i}(\omega)\right| \mid f\left(x-x_{i}+\xi_{i}(\omega) \mid\right)^{2} d x<\frac{1}{4 k}\right\} .\right.
$$


Since $V_{\omega}$ is almost surely a locally square integrable function $P(D)=1$, if we define now the set $D_{M}$ by:

$$
D_{M}=\left\{\omega ; \int_{B_{N}}\left(\sum_{|i|>M}\left|q_{i}(\omega)\right| \mid f\left(x-x_{i}+\xi_{i}(\omega) \mid\right)^{2} d x<\frac{1}{4 k}\right\},\right.
$$

then $D_{M} \subset D_{M+1}$ and $D=\bigcup D_{M}$; hence $P(D)=\lim _{M \rightarrow \infty} P\left(D_{M}\right)$ which implies that there exists an integer $M_{2}$ such that $P\left(D_{M}\right) \geqq \frac{1}{2}$. Let now $M=\max \left\{M_{1}, M_{2}\right\}$ and take $\omega \in D_{M}$; we can then estimate $\int_{B_{N}}\left|W(x)-V_{\omega}(x)\right|^{2} d x$ as follows:

$$
\begin{aligned}
\int_{B_{N}} \mid & W(x)-\left.V_{\omega}(x)\right|^{2} d x \\
\leqq & 4 \int_{B_{N}}\left|\sum_{|i| \geqq M} c_{i} f\left(x-x_{i}+u_{i}\right)\right|^{2} d x \\
& \quad+4 \int_{B_{N}} \mid \sum_{|i| \geqq M} q_{i}(\omega) f\left(x-x_{i}+\left.\xi_{i}(\omega)\right|^{2} d x\right. \\
& +2 \int_{B_{N}} \mid \sum_{|i| \leqq M} c_{i}\left(f\left(x-x_{i}+u_{i}\right)-f\left(x-x_{i}+\xi_{i}(\omega)\right)\right) \\
& +\left.\left(c_{i}-q_{i}(\omega)\right) f\left(x-x_{i}+\xi_{i}(\omega)\right)\right|^{2} d x \\
\leqq & \frac{1}{2 k}+2 C_{2 M} \sum_{|i| \leqq M} c_{i}^{2} \int_{B_{N}} \mid f\left(x-x_{i}+u_{i}\right)-f\left(x-x_{i}+\left.\xi_{i}(\omega)\right|^{2} d x\right. \\
& +2 C_{2 M} \sup _{\mid i \leqq M}\left|q_{i}(\omega)-c_{i}\right|^{2} \sum_{|i| \leqq M} \int_{\mathbb{R}^{d}} \mid f\left(x-x_{i}+\left.\xi_{i}(\omega)\right|^{2} d x,\right.
\end{aligned}
$$

where $C_{2 M}$ is a positive constant depending only on $M$.

The last term of (18) can be chosen with positive probability less than $1 / 4 k$ since by assumption $c_{i} \in \operatorname{supp} p_{q}$ and the $q_{i}$ 's are independent, and since $f \in L^{2}\left(\mathbb{R}^{d}\right)$ because $f \in L_{\text {loc }}^{2}\left(\mathbb{R}^{d}\right)$ and furthermore $f$ is such that $\sum_{|i|>K_{0}} \sup _{x \in C_{0}}\left|f\left(x-x_{i}\right)\right|<\infty$.

For the estimate of the first term of (18) we need the following remark:

Since the map $x \rightarrow U_{x}\left(U_{x} f(z)=f(z+x)\right)$ is strongly continuous from $\mathbb{R}^{d}$ into the bounded operators on $L^{2}\left(\mathbb{R}^{d}\right)$, for any $\varepsilon$, there exists a $\delta(\varepsilon, x, f)$ such that:

$$
\int_{B_{N}}|f(x+z)-f(z+y)|^{2} d z<\infty
$$

for any $y$ such that $|x-y|<\delta(\varepsilon, x, f)$. Thus in the first term of (18) if we make $\left|u_{i}-\xi_{i}(\omega)\right|$ small enough, which again is possible with positive probability since $u_{i} \in \operatorname{supp} P_{\xi}$ and the $\xi_{i}$ are independent (and also independent of the $q_{i}$ ), we have:

$$
2 C_{2 M} \sum_{|i|<M} c_{i}^{2} \int_{B_{N}}\left|f\left(x-x_{i}+u_{i}\right)-f\left(x-x_{i}+\xi_{i}(\omega)\right)\right|^{2} d x<\frac{1}{4 k} .
$$

Hence with positive probability we have that:

$$
\int_{B_{N}}\left|W(x)-V_{\omega}(x)\right|^{2} d x<\frac{1}{k}
$$

i.e. $P\left(C_{N}, k\right)>0$ hence $P\left(A_{N, k}\right)=1$. 


\section{Remarks.}

1) It is not difficult to give an alternative proof of the fact that $\sigma\left(H_{\omega}\right)$ is a nonrandom set by using the above lemma. In particular one can show that for each $\omega \in A \quad \sigma\left(H_{\omega}\right)=\Sigma$.

2) Each $\lambda \in \sum$ can be obtained by the construction in the theorem. On the other hand we can ensure that $x_{0}(N, k, \omega)$ goes to infinity as $N$ goes to infinity. Thus the sequence $\left\{\psi_{k}\right\}$ can be chosen as weakly convergent to zero and moreover orthogonal. Again by the Weyl criterion this means that each spectral value $\lambda \in \Sigma$ belongs to the essential spectrum of $H_{\omega}$.

By the previous theorem the spectrum of $H_{\omega}$ looks very large because the class of admissible potentials is very large. But the following theorem tells us that for knowing the spectrum $\Sigma$ it is enough to know the spectra of all periodic admissible potentials.

Denote by $P$ the class of all admissible potentials which are also periodic in the sense that $W \in P$ if there exists a basis $\left\{a_{i}\right\}_{i=1}^{d}$ of $\mathbb{R}^{d}$ such that

$$
W\left(x+a_{i}\right)=W(x) \quad \forall x \in \mathbb{R}^{d} .
$$

Theorem 4. In the hypotheses of Theorem 3: $\Sigma=\overline{\bigcup_{W \in P} \sigma(-\Delta+W)}$

Proof. It will be enough to show the following: if $W$ is an admissible potential, $W \in A$, then there exists a sequence of periodic admissible potentials $W_{n} \in P$ such that $-\Delta$ $+W_{n} \rightarrow-\Delta+W$ in the sense of strong resolvent convergence. From this it follows that $\bigcup_{n \in \mathbb{N}} \sigma\left(-\Delta+W_{n}\right) \supset \sigma(-\Delta+W)$ (see e.g. Reed-Simon I, VIII $2 \mathrm{~h}$ [20]). But since the union of the spectra of all the admissible potentials contains $\Sigma$ we have $\overline{\bigcup_{W \in P} \sigma(-\Delta+W)} \supset \Sigma$; but from Theorem 3 we know that $\bigcup_{W \in P} \sigma(-\Delta+W) \subset \Sigma$ so that $\Sigma=\bigcup_{W \in P} \sigma(-\Delta+W)$.

We now prove that any operator $H=-\Delta+W, W \in A$, is the strong resolvent limit of operators $H_{N}=-\Delta+W_{N}, W_{N} \in P$ for any $N \in \mathbb{N}$. Since by definition $C_{0}^{\infty}\left(\mathbb{R}^{d}\right)$ is a core for $-\Delta+W$ for any $W \in A$, it suffices to show that there are periodic potentials $W_{n} \in P$ such that $-\Delta+W_{n}$ converges strongly on $C_{0}^{\infty}\left(\mathbb{R}^{d}\right)$ to $-\Delta+W$, $W \in A$. For this it is enough to show that for any compact set $K$ and any $\varepsilon>0$ there exists a periodic admissible potential $\bar{W}$ such that:

$$
\left[\int_{K}|W(x)-\bar{W}(x)|^{2} d x\right]^{1 / 2}<\varepsilon .
$$

Since any compact set $K \subset \mathbb{R}^{d}$ can be covered by a finite number of unit cubes, it is enough to check (19) with $K=C_{0}$. Let $W(x)=\Sigma c_{i} f\left(x-x_{i}+u_{i}\right)$ be given and define

$$
\begin{array}{lll}
\tilde{c}_{i}=\tilde{c}_{i+M_{1} k}=c_{i} & \text { for }|i|<M_{1} & k \in \mathbb{Z}^{d}, \\
\tilde{u}_{i}=\tilde{u}_{i+M_{1} k^{\prime}}=u_{i} & \text { for }|i|<M_{1} & k^{\prime} \in \mathbb{Z}^{d},
\end{array}
$$

where $M_{1}$ is a positive constant such that 


$$
\sum_{|i|>M_{1}}\left|c_{i}\right| \sup _{x \in c_{0}}\left|f\left(x-x_{i}+u_{i}\right)\right|<\varepsilon / 3 .
$$

This constant always exists since $W$ is admissible. Without loss of generality we can assume $M_{2}$ greater than $N_{0}+1$, where $N_{0}$ is such that $\left|\xi_{i}\right|<N_{0}$ almost surely (see Remark after Lemma 1). Clearly the potential $\bar{W}=\Sigma \tilde{c}_{i} f\left(x-x_{i}+\tilde{u}_{i}\right)$ is periodic of period $M_{1}$. Furthermore since $\left|\tilde{u}_{i}\right|$ is uniformly bounded by $N_{0}$ (see Remark after Lemma 1) by assumption (1) on $f$, it follows that there exists a constant $M_{2}$ such that:

$$
\sup _{|i| \leqq M_{1}}\left|c_{i}\right| \sum_{|i|>M_{2}} \sup _{x \in C_{0}}\left|f\left(x-x_{i}\right)\right|<\varepsilon / 3
$$

Define

$$
\begin{array}{ll}
\bar{c}_{i}=\bar{c}_{i+M_{2} k}=\min \left\{c_{i}, \tilde{c}_{i}\right\} & \text { for }|\mathrm{i}|<M_{1} \quad k \in \mathbb{Z}^{d}, \\
\bar{u}_{i}=\bar{u}_{i+M_{2} k^{\prime}}=u_{i} & \text { for }|i|<M_{1} \quad k^{\prime} \in \mathbb{Z}^{d},
\end{array}
$$

and let $\bar{W}=\sum \bar{c}_{i} f\left(x-x_{i}+\bar{u}_{i}\right)$. Then

$$
\begin{aligned}
& \left(\int_{c_{0}}|W(x)-\bar{W}(x)|^{2} d x\right)^{1 / 2} \\
& \leqq \sup _{x \in C_{0}}\left|\sum_{|i|>M_{1}}\left(c_{i} f\left(x-x_{i}+u_{i}\right)-\bar{c}_{i} f\left(x-x_{i}+\bar{u}_{i}\right)\right)\right| \\
& \leqq \\
& \quad \sum_{|i|>M_{1}}\left|c_{i}\right| \sup _{x \in C_{0}}\left|f\left(x-x_{i}+u_{i}\right)\right|+ \\
& \quad+\sum_{|i|>M_{2}}\left|\bar{c}_{i}\right| \sup _{x \in C_{0}}\left|f\left(x-x_{i}+u_{i}\right)\right| \\
& \quad+\sum_{M_{1}<|i| \leqq M_{2}}\left|\bar{c}_{i}\right| \sup _{x \in C_{0}}\left|f\left(x-x_{i}+u_{i}\right)\right| \\
& <\frac{\varepsilon}{3}+\frac{\varepsilon}{3}+\sum_{M_{1} \leqq|i|<M_{2}}\left|c_{i}\right| \sup _{x \in \mathcal{C}_{0}}\left|f\left(x-x_{i}+\bar{u}_{i}\right)\right|<\varepsilon .
\end{aligned}
$$

Remark 1. From the above theorem it follows that in principle the spectrum of the random operator $H_{\omega}$ has band structure, i.e. the set $\Sigma$ is a union of closed intervals with possibly gaps between them.

Remark 2. The spectrum of a possible pure crystal (i.e. $q_{i}=\bar{q}, \xi_{i}=\bar{\xi}$ for any $i \in \mathbb{Z}^{d}$ ) is always contained in the spectrum of the random mixture. For example if $0 \in \operatorname{supp} P_{q_{0}}$, then the set $[0,+\infty[\subset \Sigma$.

Remark 3. The spectrum $\Sigma$ depends only on the support of the distribution $P_{q_{0}}$ of each $q_{i}$. For a similar result in the discrete case (when the Hilbert space is $l^{2}\left(\mathbb{Z}^{d}\right)$ ) see Kunz-Souillard [13].

Remark 4. Actually the random variables $q_{i}$ and $\xi_{i}$ need not to be independent; it is enough that they form a stationary metrically transitive random field such that the support of the conditional distribution $P\left(q_{0} \mid q_{i} i \neq 0\right)$ of $q_{0}$ given $q_{i}(i \neq 0)$ is equal to the support of $P\left(q_{0}\right)$ and the same for the $\xi_{i}$.

\section{Section 3}

In this section we give a simple condition for the existence of gaps 
in the spectrum of the random operator $H_{\omega}=-\Delta+\sum q_{i}(\omega) f\left(x-x_{i}\right)$ on $L^{2}\left(\mathbb{R}^{d}\right)$, which is useful when dealing with concrete examples. Let $W(x)=$ $\sum \lambda_{i} f\left(x-x_{i}\right)$ be a periodic admissible potential and let $\left\{a_{i}\right\}_{i=1}^{d}$ be a basic of the vector space $\mathbb{R}^{d}$ such that

$$
W\left(x+a_{i}\right)=W(x) \quad \forall x \in \mathbb{R}^{d} .
$$

Clearly the Hamiltonian $H_{w}=-\Delta+w$ is invariant under the group $A=\left\{n a_{i}\right.$, $\left.n \in \mathbb{Z}^{d}\right\}$, so that it can be decomposed as follows:

$$
H_{w}=\int_{\hat{\Lambda}} H_{\omega}(k) d k=\frac{1}{|B|} \int_{B} H_{w}(k) d k,
$$

where $\hat{A}$ is the dual group of $A$ and $B$ is the Brillouin zone (see e.g. Reed-Simon IV [20] and Avron-Grossmann-Rodriguez [3]. $H_{\omega}(k)$ are called the reduced Block Hamiltonians; from the above integral decomposition it follows that the spectrum of $H_{w}$ is the union of the spectra $H_{w}(k)$. Furthermore, since $W(x) \in L_{\text {loc }}^{2}\left(\mathbb{R}^{d}\right)$, it follows that for $d \leqq 3$ the reduced Hamiltonians have compact resolvent and thus their spectrum consists only of isolated eigenvalues $E_{n}(W, k)$, labeled by the discrete parameter $n \in \mathbb{N}$, of finite multiplicity (see Avron-Grossmann-Rodriguez, Th. 3, 1 [3]).

Assumption $A$. The eigenvalues $E_{n}(W, k)$ of the reduced Hamiltonians $H_{W}(k)$, $W=\sum \lambda_{i} f\left(x-x_{i}\right)$, are such that for any $n \in \mathbb{N}$ and any $k \in B$ there exist two numbers $\underline{\lambda}^{(n, k)}, \bar{\lambda}^{(n, k)}, \lambda_{\text {min }} \leqq \underline{\lambda}^{(n, k)} \leqq \lambda_{i} \leqq \bar{\lambda}^{(n, k)} \leqq \lambda_{\text {max }}$, where $\lambda_{\text {min }}\left(\lambda_{\max }\right)$ is the inf (sup) of the set supp $P_{q_{0}}, P_{q_{0}}$ being the distribution function of each of the random variables $q_{i}$, or $\lambda_{\text {min }}=-\infty\left(\lambda_{\max }=+\infty\right)$ if the inf (sup) of supp $P_{q_{0}}$ does not exist, such that:

$$
\begin{aligned}
& E_{n}(\underline{W}, k) \leqq E_{n}(W, k) \leqq E_{n}(\bar{W}, k), \text { or } \\
& E_{n}(\underline{W}, k) \geqq E_{n}(W, k) \geqq E_{n}(\bar{W}, k), \\
& \text { with } \underline{W}(x)=\underline{\lambda}^{(n, k)} \sum f\left(x-x_{i}\right), \bar{W}(x)=\bar{\lambda}^{(n, k)} \sum f\left(x-x_{i}\right) .
\end{aligned}
$$

Remark. By the mini-max principle Assumption A holds if for example $f(x)$ has a definite sign.

Theorem 5. If Assumption $A$ is satisfied and the open interval $(\alpha, \beta)$ does not belong to the spectrum of the periodic Hamiltonian $-\Delta+\lambda \Sigma f\left(x-x_{i}\right)$ for any $\lambda_{\min } \leqq \lambda \leqq \lambda_{\max }$, then $(\alpha, \beta) \bigcap \Sigma=\varnothing$, where $\Sigma$ is the spectrum of $H_{\omega}=-\Delta+\sum q_{i}(\omega) f\left(x-x_{i}\right)$ almost surely.

Proof. By Theorem 4 it is enough to show that $(\alpha, \beta) \bigcap \sigma(-\Delta+W) \equiv D=\varnothing$ for any periodic admissible potential $W$. Assume $D \neq \varnothing$ and let $E_{0} \in D$. By the integral decomposition of $-\Delta+W$ it follows that $E_{0}=E_{n_{0}}\left(W, k_{0}\right)$ for some $n_{0} \in \mathbb{N}$ and $k_{0} \in B$. By assumption $A$ we have

$$
E_{n_{0}}\left(W, k_{0}\right) \leqq E_{n_{0}}\left(W, k_{0}\right) \leqq E_{n_{0}}\left(\bar{W}, k_{0}\right),
$$

for some $W(x)=\underline{\lambda}^{\left(n_{0}, k_{0}\right)} \sum_{i \in \mathbb{Z}^{d}} f\left(x-x_{i}\right)$, and

$$
\bar{W}(x)=\bar{\lambda}^{\left(n_{0}, k_{0}\right)} \sum_{i \in \mathbb{Z}^{d}} f\left(x-x_{i}\right),
$$


with $\underline{\lambda}^{\left(n_{0}, k_{0}\right)} \leqq \bar{\lambda}^{\left(n_{0}, k_{0}\right)}$ or vice versa. Since $E_{n_{0}}\left(\lambda \Sigma f\left(x-x_{i}\right), k_{0}\right)$ is a continuous function of $\lambda$, (see e.g. Reed-Simon IV [20]) there exists a $\underline{\lambda}^{\left(n_{0}, k_{0}\right)} \leqq \tilde{\lambda} \leqq \bar{\lambda}^{\left(n_{0}, k_{0}\right)}\left(\right.$ or $\underline{\lambda}^{\left(n_{0}, k_{0}\right)} \geqq \tilde{\lambda}$ $\geqq \bar{\lambda}^{\left(n_{0}, k_{0}\right)}$ ) such that

$$
E_{n_{0}}\left(W, k_{0}\right)=E_{n_{0}}\left(\tilde{W}, k_{0}\right) \text { when } \tilde{W}(x)=\tilde{\lambda} \sum_{i \in \mathbb{Z}^{d}} f\left(x-x_{i}\right) .
$$

But this means that $E_{0}=E_{n_{0}}\left(W, k_{0}\right) E_{n_{0}}\left(\tilde{W}, k_{0}\right)$ belongs to the spectrum of $-\Delta+\lambda \Sigma f\left(x-x_{i}\right)$ in contradiction with the hypothesis.

Corollary 1. If the supp $P_{q_{0}}$ is connected and if Assumption $A$ holds, then the open interval $(\alpha, \beta)$ is a gap for the random Hamiltonian $H_{\omega}$ if and only if it is a gap for all the periodic Hamiltonians $H_{\lambda}=-\Delta+\lambda \Sigma f\left(x-x_{i}\right) \lambda \in \operatorname{supp} P_{q_{0}}$.

Proof. It is a direct consequence of the previous theorem and of Theorem 4.

It follows from the above Corollary that, in some case, the study of the (possible) gaps in the spectrum $\Sigma$ of $H_{\omega}$ is reduced to the study of the gaps of the "pure crystals" $-\Delta+\sum_{i \mathbb{Z}^{d}} f\left(x-x_{i}\right)$, where $\lambda$ runs in the supp $P_{q_{0}}$. In the examples we shall consider it will turn out to be sufficient to study the gaps of the "pure crystals" corresponding to the values $\lambda=\lambda_{\max }$ and $\lambda=\lambda_{\text {min }}$.

\section{Section 4}

In this last section we give first two one-dimensional examples of random operators of the Kronig-Penney type for which infinitely many gaps occur, and then we show that also for the random version of the periodic point interaction model in three dimensions treated by Grossmann, H $\phi$ egh-Krohn, Mebkhout [9] still a gap is present in the nonrandom spectrum $\Sigma$. While the first example is only an application of the general result of Sects. 1-3, the other two examples require new proofs of results similar to that of the previous sections, since the above theorems cannot be applied directly because of the strong singularities of these Hamiltonians.

1) The Kronig-Penney model with a step potential in one dimension (see Kronig-Penney [12]).

Let $f(x)=\chi_{[0, a]}(x), 0<a<x_{1}\left(\chi_{A}(\cdot)\right.$ is the characteristic function of the set $\left.A \subset \mathbb{R}\right)$ and let $V(x)=\Sigma f\left(x-x_{i}\right)$ when the lattice $\left\{x_{i}\right\}_{i \in \mathbb{Z}}$ is assumed for simplicity to be $\mathbb{Z}$. Furthermore let $\left\{q_{i}\right\}_{i \in \mathbb{Z}}$ be independent, identically distributed random variables such that:

$$
0<M_{1} \leqq q_{i}(\omega) \leqq M_{2}<\infty \quad \forall \omega \in \Omega, \forall i \in \mathbb{Z} .
$$

and define $V_{\omega}(x)=\sum_{i \in \mathbb{Z}} q_{i}(\omega) f\left(x-x_{i}\right)$.

Propositional 1. Denote by $\Sigma$ the almost surely constant spectrum of $H_{\omega}=$ $-d^{2} / d x^{2}+V_{\omega}$. Then, for each of the points $E_{n}=\left(n \pi /\left(x_{1}-a\right)\right)^{2}, E_{n}<M_{1}$, there exists a neighborhood $A_{n}$ such that $A_{n} \cap \Sigma=\varnothing$.

Proof. In Flügge [6] it is shown that for any $0<\lambda<+\infty$ the Hamiltonian 
$-d^{2} / d x^{2}+\lambda \sum_{i \in \mathbb{Z}} f\left(x-x_{i}\right)$ has gaps $A_{n}^{\lambda}$ around the points $E_{n}<\lambda$. If we define $A_{n}=$ $\bigcap_{M_{1} \leqq \lambda \leqq M_{2}} A_{n}^{\lambda}$ we have that $A_{n} \neq \varnothing$ and that $A_{n}$ is a common gap for all the Hamiltonian $-d^{2} / d x^{2}+\lambda \sum_{i \in \mathbb{Z}} f\left(x-x_{i}\right)$ with $M_{1} \leqq \lambda \leqq M_{2}$. Furthermore since $f(x)$ has a definite sign $(f(x) \geqq 0)$ and $f(x) \leqq 1$ Assumption A holds so that we get the statement by Theorem 5 .

Remark. Let $A_{n}^{\lambda} \equiv\left(\alpha_{n}^{\lambda}, \beta_{n}^{\lambda}\right)$ be the gap around the point $\left(n \pi /\left(x_{i}-a\right)\right)^{2}$ for the periodic Hamiltonian $-d^{2} / d x^{2}+\lambda \sum_{i \in \mathbb{Z}} f\left(x-x_{i}\right) M_{1} \leqq \lambda \leqq M_{2}$. By the general theory of onedimensional periodic Schrödinger operators (see, e.g. Reed-Simon IV [20]), we know that $\alpha_{n}$ and $\beta_{n}$ are then $n^{\text {th }}$ eigenvalues of $-d^{2} / d x^{2}+\lambda \sum_{i \in \mathbb{Z}} f\left(x-x_{i}\right)$ on $L^{2}\left(\left[0 x_{1}\right], d x\right)$ with respectively periodic and antiperiodic boundary conditions if $n$ is even and vice versa if $n$ is odd. Hence, by the mini-max principle $\alpha_{n}^{\lambda}, \beta_{n}^{\lambda}$ are monotone increasing functions of $\lambda$, so that the gap $A_{n}$ for the random operator $-d^{2} / d x^{2}$ $+\Sigma q_{i}(\omega) f\left(x-x_{i}\right)$ is given by: $A_{n}=\left(\alpha_{n}^{M_{2}}, \beta_{n}^{M_{1}}\right)$.

2) We now pass to consider a random Kronig-Penney model with point interactions formally defined by:

$$
H_{\omega}=-\frac{d^{2}}{d x^{2}}+\sum_{i \in \mathbb{Z}} q_{i}(\omega) \delta\left(x-x_{i}\right),
$$

where $\left\{q_{i}(\omega)\right\}_{i \in \mathbb{Z}}$ are independent identically distributed random variables satisfying $0<c_{1} \leqq q_{i}(\omega) \leqq c_{2}<+\infty$ for any $i \in \mathbb{Z}$ and $\omega \in \Omega$. The Hamiltonian $H_{\omega}$ can be well defined as a sum of quadratic forms as follows: Denote by $Q=Q\left(-d^{2} / d x^{2}\right)$ the form domain of the operator $-d^{2} / d x^{2}$ on $L^{2}(\mathbb{R})$ and by $Q_{0}$ the function in $Q$ with compact support. We define on $Q_{0}$ the quadratic form:

$$
\beta_{\omega}(\varphi, \psi)=\sum_{i \in \mathbb{Z}} q_{i}(\omega) \varphi\left(x_{i}\right) \psi\left(x_{i}\right) .
$$

First we show: For any $a>0$, there is a $b \in \mathbb{R}$ such that:

$$
\left|\beta_{\omega}(\psi, \psi)\right| \leqq a\left\langle\psi^{\prime}, \psi^{\prime}\right\rangle+b\langle\psi, \psi\rangle
$$

for any $\psi \in Q_{0}$ and $\omega \in \Omega$. In order to prove this, take a sequence $\left\{\psi_{l}\right\}_{i \in \mathbb{Z}}$ in $C_{0}^{x}(\mathbb{R})$ such that:

1) $\left.\operatorname{supp} \psi_{i} \subset\right] x_{i-1}, x_{i+1}[$,

2) $\sum_{i \in \mathbb{Z}} \psi_{i}^{2}(x)=1$,

3) $\sup _{x \in \mathbb{R}} \sum_{i \in \mathbb{Z}}\left|\psi_{i}^{\prime}(x)\right|^{2}<\infty$.

A sequence satisfying 2) and 3) is called a "local partition" in Morgan [16]. 
We have:

$$
\left|\beta_{\omega}\left(\psi_{i} \psi, \psi_{i} \psi\right)\right| \leqq c_{2}\left|\psi\left(x_{i}\right)\right|^{2} \leqq a\left\langle\left(\psi_{i} \psi\right)^{\prime},\left(\psi_{i} \psi\right)^{\prime}\right\rangle+b\langle\psi, \psi\rangle
$$

(see e.g. Reed-Simon II, X. 2[20]). By repeating now word by word the proof of Theorem 2.2 in Morgan [16] we get from the local estimate (23) the global one (22).

Now we show that $\beta_{\omega}$ can be defined on the whole space $Q\left(-d^{2} / d x^{2}\right)$. Let $\psi \in Q$, choose $\psi_{n} \in Q_{0}, \psi_{n} \rightarrow \psi$ in $L^{2}(\mathbb{P}), \psi_{n}^{\prime} \rightarrow \psi^{\prime}$ in $L^{2}(\mathbb{R})$, which is possible since $Q_{0}$ is dense in $Q$ with respect to the norm $\left(\left\|\psi^{\prime}\right\|^{2}+\|\psi\|^{2}\right)^{1 / 2} ;$ moreover we can choose $\psi_{n}$ in such a way that $\psi_{n}(x)=\psi(x)$ for $|x| \leqq n$. Now:

$$
\begin{aligned}
\sum_{|i| \leqq n} q_{i}(\omega)\left|\psi\left(x_{i}\right)\right|^{2} & =\sum_{|i| \leqq n} q_{i}(\omega)\left|\psi_{n}\left(x_{i}\right)\right|^{2} \leqq\left. C_{2} \sum_{i \in \mathbb{Z}} \psi_{n}\left(x_{i}\right)\right|^{2} \\
& \leqq a\left\|\psi_{n}^{\prime}\right\|^{2}+b\left|\psi_{n}\right|^{2} .
\end{aligned}
$$

The last expression is bounded independent of $n$, since $\psi_{n}^{\prime}$ and $\psi_{n}$ are convergent, hence the norms are bounded. Thus $\beta_{\omega}(.,$.$) is well defined on Q\left(-d^{2} / d x^{2}\right)$.

Furthermore, by continuity inequality (22) holds for all $\psi \in Q$. Hence we can apply the KLMN (Kata, Lax, Milgram, Nelson)-Theorem (see e.g. Reed-Simon II, X. 2 [20]) to get a well defined, unique selfadjoint operator $H_{\omega}$ on $L^{2}(\mathbb{R})$ associated with the closed quadratic form $\gamma_{\omega}(\psi, \psi):=\left\langle\psi^{\prime}, \psi^{\prime}\right\rangle+\beta_{\omega}(\psi, \psi)$ on $Q\left(-d^{2} / d x^{2}\right)$. It is this operator we mean by the formal expression in (21).

In [11] we showed that the spectrum of $H$ is a nonrandom set. We call this set $\Sigma$. In order to investigate $\Sigma$ we have to prove analogs of Theorems 3 and 4 for the (very singular) Hamiltonian $H_{\omega}$. Although it is possible to give proofs similar to those of Sect. 2, since $H_{\omega}$ can be shown to be essentially selfadjoint on a set of functions with compact supports, we prefer to give a more direct proof based on the explicit expression of the resolvent of $H_{\omega}$. This method has the advantage that it can be extended immediately to the three-dimensional case (see the next example) and moreover the case of continuously distributed random variables $\left\{q_{i}\right\}_{i \in \mathbb{Z}}$ causes no further difficulty as it does for the previous method.

We call, as in Sect. 2, the formal expression $W=\Sigma \lambda_{i} \delta\left(x-x_{i}\right)$ an admissible potential if $\lambda_{i} \in \operatorname{supp} P_{q_{0}}$ for all $i \in \mathbb{Z}$. In this case we also call the sequence $\left\{\lambda_{i}\right\}_{i \in \mathbb{Z}}$ admissible.

Now we give explicitly the resolvent of the operator

$$
H_{\left\{\lambda_{1}\right\}}=-\frac{d^{2}}{d x^{2}}+\sum_{i \in \mathbb{Z}} \lambda_{i} \delta\left(.-x_{i}\right) \lambda_{i} \text { bounded. }
$$

Faris [5] (in §5) computes the resolvent of the operator $H_{\lambda_{0}}=-\frac{d^{2}}{d x^{2}}+\lambda_{0} \delta($.$) .$ The resolvent is for $E \notin \sigma\left(-\frac{d^{2}}{d x^{2}}\right):\left(H_{\lambda_{0}}-E\right)^{-1} \psi(x)=\int_{\mathbb{R}} G_{E}(x-y) \psi(y) d y$ $-\left(\frac{1}{\lambda_{0}}+\frac{1}{2 \sqrt{E}}\right)^{-1} \int_{\mathbb{R}} G_{E}(y) \psi(y) d y G_{E}(x)$, where $G_{E}(x)=\frac{1}{2 \sqrt{-E}} e^{-\sqrt{-E}|x|}$ is the free Green's function in one dimension 
and $\sqrt{-E}$ is chosen such that $\operatorname{Re}(\sqrt{-E})>0$. In the case of finitely many $\delta$ 's we get a similar expression:

$$
\begin{gathered}
\left(-\frac{d^{2}}{d x^{2}}+\sum_{i=-n}^{n} \lambda_{i} \delta\left(x-x_{i}\right)-E\right)^{-1} \psi(x)=\int_{\mathbb{R}} G_{E}(x-y) \psi(y) d y \\
-\sum_{i, j=-n}^{n}\left(T_{E}^{(n)-1}\right)_{i j} \int G_{E}\left(y-x_{j}\right) \psi(y) d y G_{E}\left(x-x_{i}\right),
\end{gathered}
$$

where $T_{E}^{(n)}$ is the $n \times n$-matrix given by $\left(T_{E}^{(n)}\right)_{i j}=\delta_{i j} / \lambda_{i}+G_{E}\left(x_{i}-x_{j}\right)$. This expression as well as its calculation is in complete analogy with those given in Grossmann, H $\phi$ egh-Krohn and Mebkhout [9] where a three-dimensional point interaction is considered.

Using Theorem 7-10 in Faris [5] we get easily that $-\frac{d}{d x^{2}}+\sum_{i=n}^{n} \lambda_{i} \delta\left(.-x_{i}\right)$ converges in the strong resolvent sense to $H_{\left\{\lambda_{i}\right\}}=-\frac{d^{2}}{d x^{2}}+\sum_{i=-\infty}^{+\infty} \lambda_{i} \delta\left(.-x_{i}\right)$ as $n$ goes to infinity.

Hence the resolvent of $H_{\left\{\lambda_{t}\right\}}$ is given by:

$$
\begin{aligned}
\left(H_{\left\{\lambda_{i}\right\}}-E\right)^{-1} \psi(x)= & \int G_{k}(x-y) \psi(y) d y-\sum_{i . j \in \mathbb{Z}}\left(T_{E}^{-1}\right)_{i j} \\
& \int G_{E}\left(y-x_{j}\right) \psi(y) d y G_{E}\left(x-x_{i}\right),
\end{aligned}
$$

where $T_{E}$ is the bounded operator on $l^{2}(\mathbb{Z})$ given by: $\left(T_{E}\right)_{i j}=\delta_{i j} / \lambda_{i}+G_{E}\left(x_{i}-x_{j}\right)$. Indeed, that (24) is the correct limit for the weak resolvent convergence is easily obtained by computation observing that $\psi_{i}=\int G_{E}\left(x-x_{j}\right) \psi(x) d x$ is in $l^{2}(\mathbb{Z})$ that $T_{E}$ and $T_{F}^{(n)}$ can be looked upon as bounded operators in $l^{2}(\mathbb{Z})$ and $T_{E}^{(n)-1} \rightarrow T_{E}^{-1}$ weakly in $l^{2}(\mathbb{Z})$ as $n \rightarrow \infty$. But since we know that $-\frac{d^{2}}{d x^{2}}+\sum_{i=-n}^{n} \lambda_{i}\left(-x_{i}\right)$ converges in the strong resolvent sense, the operator in (24) is also the strong limit.

Proposition 2 Let $W$ be an admissible potential, then $\sigma(-\Delta+W) \subset \Sigma$.

Proof. The proof is based on the following lemma:

Lemma 3. Let $\operatorname{Im} E \neq 0$ and let $W=\left\{\lambda_{i}\right\}_{i \in \mathbb{Z}}$ be an admissible potential. Then there exists a sequence of $\omega_{n} \in \Omega_{1}$, when $\Omega_{1}=\left\{\omega \in \Omega ; \sigma\left(H_{\omega}\right)=\Sigma\right\}$, such that the operator $T_{E}\left(\omega_{n}\right)$ on $l_{2}(\mathbb{Z})$ with matrix elements

$$
\frac{1}{q_{k}\left(\omega_{n}\right)} \delta_{k j}+G_{E}\left(x_{k}-x_{j}\right)
$$

converges in the strong resolvent sense to the operator $T_{E}$ given by

$$
\frac{1}{\lambda_{k}} \delta_{k j}+G_{E}\left(x_{k}-x_{j}\right)
$$

Proof. Define $\Omega_{n}=\left\{\omega \in \Omega ;\left|q_{i}(\omega)-\lambda_{i}\right|<1 / n \quad \forall|i| \leqq n\right\}$. 
Since $P\left(\Omega_{1}\right)=1$ and $P\left(\Omega_{n}\right)>0, \Omega_{1} \bigcap \Omega_{n} \neq \varnothing$.

Hence we can pick $\omega_{n} \in \Omega_{n} \bigcap \Omega_{1}$ and compute for $\psi \in l_{2,0}(\mathbb{Z})=\left\{\psi \in l_{2}(\mathbb{Z}) ; \psi(i) \neq 0\right.$ only for finitely many $i \in \mathbb{Z}\}$ the quantity; $\left\|\left(T_{E}\left(\omega_{n}\right)-T_{E}\right) \psi\right\|_{l_{2}}(\mathbb{Z})$.

We have:

$$
\begin{aligned}
& \sum_{k \in \mathbb{Z}}\left|\sum_{j \in \mathbb{Z}}\left[\frac{1}{q_{k}\left(\omega_{n}\right)} \delta_{k j}+G_{E}\left(x_{k}-x_{j}\right)\right]-\left(\frac{1}{\lambda_{k}} \delta_{k j}+G_{E}\left(x_{k}-x_{j}\right)\right) \psi(j)\right|^{2} \\
& =\sum_{k \in \mathbb{Z}}\left|\left(\frac{1}{q_{k}\left(\omega_{n}\right)}-\frac{1}{\lambda_{k}}\right) \psi(k)\right|^{2} .
\end{aligned}
$$

By definition of $\omega_{n}$ and the fact that $\psi \in l_{2},(\mathbb{Z})$ the last expression goes to zero as $n \rightarrow \infty$. Since $T_{E}\left(\omega_{n}\right)$ and $T_{E}$ are bounded and symmetric, it follows that $T_{E}\left(\omega_{n}\right) \rightarrow T_{E}$ in the strong resolvent sense.

Now we give the proof of the proposition:

Proof of the Proposition. First we note that the $p$-space version of the resolvent of $H_{\left\{\lambda_{t}\right\}}$ is given by:

$$
\left(H_{\left\{\lambda_{t}\right\}}-E\right)^{-1} \psi(p)=\frac{1}{p^{2}-E} \psi(p)+\frac{1}{2 \pi} \sum_{j, k \in \mathbb{Z}}\left(T_{E}\right)_{k j}^{-1} \frac{1}{2 \pi} \int \frac{\psi(q)}{q^{2}-E} e^{-i x_{j} q} d q \frac{1}{p^{2}-E} e^{i x_{k} p} .
$$

Take now $\psi \in C_{0}^{\infty}(R)$, then $\tilde{\psi}(j):=\frac{1}{2 \pi} \int_{\mathbb{R}} \frac{\psi(q)}{q^{2}-E} e^{-\imath q x} d q$ is an element of $l_{2}(\mathbb{Z})$. By the previous lemma: $\left(T_{E}^{-1}-T_{E}\left(\omega_{n}\right)^{-1}\right) \widetilde{\psi}=: \tilde{\psi}_{n}$ tends to zero in $l_{2}(\mathbb{Z})$-norm. The Fourier transform $\varphi_{n}(q)=(1 / 2 \pi) \Sigma \widetilde{\psi}_{n}(j) e^{i x_{j} q}$ of $\widetilde{\psi}_{n}$ hence tends to zero as a function in $L^{2}(0,2 \pi)$. But

$$
\int_{\mathbb{R}} \frac{\left|\varphi_{n}(p)\right|^{2}}{\left(p^{2}-E\right)^{2}} d p \leqq\left\|\varphi_{n}\right\|_{L^{2}(0,2 \pi)}\left\|\frac{1}{p^{2}-E}\right\|_{L^{2}(\mathbb{R})} \rightarrow 0
$$

we have proved that $H_{\omega(n)} \rightarrow H_{\omega}$ in the strong resolvent sense. From this we get $\sigma\left(H_{\omega}\right) \subset \overline{\bigcup \sigma\left(H_{\omega(n)}\right)}=\Sigma$ since $\omega(n) \in \Omega_{1}$ for all $n \in N$.

Call now $W=\sum \lambda_{i} \delta\left(.-x_{i}\right)$ an admissible periodic potential if $\lambda_{i+M}=\lambda_{i}$ for some $M \in \mathbb{Z}, \lambda_{i} \in \operatorname{supp} P_{q_{0}}$. As in Sect. 2 we denote by $P$ the class of periodic admissible potentials.

\section{Proposition 3.}

$$
\Sigma=\overline{\bigcup_{W \in P} \sigma\left(H_{W}\right)}
$$

Proof. As before we need a preparatory lemma:

Lemma 4. Let $W=\left\{\lambda_{i}\right\}_{i \in \mathbb{Z}}$ be an admissible periodic potential. Then there exist $W_{n}=\left\{\lambda_{k}^{(n)}\right\}_{k \in \mathbb{Z}} \in P$ such that $T_{E}\left(W_{n}\right) \rightarrow T_{E}(W)$ in the strong resolvent sense in $l_{2}(\mathbb{Z})$, 
where $T_{E}\left(W_{n}\right)$ and $T_{E}(W)$ have respectively matrix elements:

$$
\frac{1}{\lambda_{k}^{(n)}} \delta_{k j}+G_{E}\left(x_{k}-x_{j}\right) \text { and } \frac{1}{\lambda_{k}} \delta_{k j}+G_{E}\left(x_{k}-x_{j}\right) .
$$

Proof. Choose $\lambda_{k}^{(n)}=\lambda_{k}$ if $|k| \leqq n$ and $\lambda_{k+2 n+1}^{(n)}=\lambda_{k}^{(n)} \quad \forall n \in \mathbb{Z}$.

Then, as in Lemma 3 it can be shown immediately that:

$$
\left\|\left[T_{E}\left(W_{n}\right)-T_{E}(W)\right] \psi\right\|_{l_{2}(\mathbb{Z})} \rightarrow 0 \text {, as } n \rightarrow \infty \quad \forall \psi \in l_{2},(\mathbb{Z}) .
$$

From the above lemma, precisely as in the previous proposition, we get that $H_{W_{n}} \rightarrow H_{W}$ in the strong resolvent sense. This implies that $\sigma\left(H_{W}\right) \subset \bigcup \sigma\left(H_{W_{n}}\right)$, i.e. $\Sigma \subset \bigcup_{w \in P} \sigma\left(H_{\omega}\right)$; and by the previous proposition we get:

$$
\Sigma=\overline{\bigcup_{W \in P} \sigma\left(H_{\omega}\right)} .
$$

Now we turn to the determination of the spectrum of the operator $H$. As it was shown by Kronig and Penney [12] the spectrum of the operator $H_{\lambda}$ formally defined by

$$
H_{\lambda}=-\frac{d^{2}}{d x^{2}}+\lambda \sum_{i=-\infty}^{\infty} \delta\left(x-x_{i}\right) \text { is given by } \sigma\left(H_{\lambda}\right)=\bigcup\left[f_{n}(\lambda),(n \pi)^{2}\right],
$$

where the $f_{n}$ are continuous monotone increasing functions of $\lambda \geqq 0$ and $f_{n}(\lambda)>$ $((n-1) \pi)^{2}$ for $\lambda>0$ (see also Flügge [6]). It is a special property of this operator that $\sigma\left(H_{\lambda}\right) \subset \sigma\left(H_{\mu}\right)$ whenever $\mu \leqq \lambda$.

The reduced Bloch Hamiltonians of $H_{\left\{\lambda_{1}\right\}}=-d^{2} / d x^{2}+\Sigma \lambda_{i} \delta\left(x-x_{i}\right)$, where $\lambda_{i+N}=\lambda_{i}$ can be defined using the KLMN-theorem as the unique selfadjoint operator associated to the closed form:

$$
\left\langle H_{\left\{\lambda^{\prime}\right\}}(k) \psi, \psi\right\rangle:=\left\langle\psi^{\prime}, \psi^{\prime}\right\rangle+\sum_{i=0}^{N-1} \lambda_{i} \psi\left(x_{i}\right) \overline{\psi\left(x_{i}\right)}
$$

on the form domain $Q\left(\left(-d^{2} / d x^{2}\right)_{k}\right)$ of the Laplacian on $L^{2}-1 / 2,(N-1 / 2)$ with boundary conditions

$$
\psi\left(-\frac{1}{2}\right)=e^{i k(N)} \psi\left(N-\frac{1}{2}\right) \text { and } \psi^{\prime}\left(-\frac{1}{2}\right)=e^{i k N} \psi^{\prime}\left(W-\frac{1}{2}\right) .
$$

These reduced Hamiltonians have discrete eigenvalues (see e.g. Avron, Grossman and Rodriguez [3]) and satisfies Assumption $A$ of Sect. 3, where (i) can be checked by the mini-max-principle and (ii) follows from the computation by Kronig and Penney [12]. By repeating word by word the proof of Theorem 5 we can show that Theorem 5 holds with the Hamiltonian $-\Delta+\lambda \Sigma f\left(x-x_{i}\right)$ replaced by the Hamiltonian $-d^{2} / d x^{2}+\lambda \Sigma \delta\left(x-x_{i}\right)$. Hence we conclude that $]((n-1) \pi)^{2}, f_{n}\left(\lambda_{\text {min }}\right)[$ where $\lambda_{\text {min }}=\inf \left\{x \in \operatorname{supp} P_{q_{0}}\right\}>0$ is a gap for the spectrum $\Sigma$ of the random operator

$$
H_{\omega}:=-\frac{d^{2}}{d x^{2}}+\sum q_{i(\omega)} \delta\left(x-x_{i}\right)
$$

But from Proposition 2 we know that $\sigma\left(H_{\lambda_{\min }}\right) \subset \Sigma$. Hence we have shown: 
Proposition 4. Let $\left\{q_{i}(\omega)\right\}_{i \in \mathbb{Z}}$ be independent distributed random variables satisfying 0 $<c_{1} \leqq q_{i}(\omega) \leqq c_{2}$ and let $H_{\omega}$ be formally given by $H_{\omega}:=-d^{2} / d x^{2}+\Sigma q_{i}(\omega) \delta\left(x-x_{i}\right)$, the exact meaning of which is given above.

Then the spectrum $\Sigma$ of almost all $H_{\omega}$ is given by

$$
\Sigma=\sigma\left(H_{q_{\min }}\right)
$$

where $H_{q_{\min }}$ is formally $-d^{2} / d x^{2}+q_{\min } \Sigma \delta\left(x-x_{i}\right)$ and $q_{\min }=\inf \left\{q ; q \in \operatorname{supp} P_{q_{0}}\right\}$.

3) Random point interactions in three dimensions. As the last example we treat the random version of the point interactions model in three dimensions extensively investigated by Grossman, Hфegh-Krohn and Mebkhout [9]. For other approaches to such operators see Albeverio and Hфegh-Krohn [2] and Albeverio, Fenstad and Hфegh-Krohn [1]. Let $\left\{q_{i}(\omega)\right\}_{i \in \mathbb{Z}^{3}}$ be independent identically distributed random variables such that $\left|q_{i}(\omega)\right|<M \forall i \in \mathbb{Z}^{3}$ and $\forall \omega \in \Omega$, for some positive constant $M$, and let $H_{\omega}$ be the selfadjoint operator on $L^{2}\left(\mathbb{R}^{3}\right)$ whose resolvent is given by:

$$
\begin{aligned}
& (H-E)^{-1}=\left(p^{2}-E\right)^{-1} \sum_{k, j \mathbb{Z}^{3}}\left[\left(q_{k}(\omega)-\frac{i \sqrt{ } E}{4 \pi}\right) \delta_{k j}-\widetilde{G}_{E}(k-j)\right]_{\mathbb{Z}^{3}}^{-1} \text { and } \\
& H_{\omega}(2 \pi)^{-3} \frac{e^{i(p k-q j)}}{\left(p^{2}-E\right)\left(q^{2}-E\right)},
\end{aligned}
$$

where $\operatorname{Im} E \neq 0, \tilde{G}_{E}(k-j)=(1 / 4 \pi) e^{i \sqrt{ } E|k-j|} /|k-j|$, if $k \neq j$ and $\tilde{G}_{E}(0)=0$ and $\left[\left(q_{k}(\omega)-i \sqrt{E} / 4 \pi\right) \delta_{k j}-\tilde{G}_{E}(k-j)\right]_{\mathbb{Z}^{3}}^{-1}$ is the inverse as an operator on $l_{2}\left(\mathbb{Z}^{3}\right)$. The sum in (25) is absolutely convergent in the sense that if we integrate with respect to $L^{2}\left(\mathbb{R}^{3}\right)$ functions of $p$ and $q$ respectively, then the sum is absolutely convergent. We remark that, as in the one-dimensional case, the operator on $l_{2}\left(\mathbb{Z}^{3}\right)$ given by $q_{k}(\omega) \delta_{k j}$ $-\widetilde{G}_{E}(k-j)$ is a bounded operator if $\operatorname{Im} E \neq 0$.

In our previous paper [11] we proved that the spectrum of $H$ is almost surely a nonrandom set $\Sigma$ of the real line. As in the one-dimensional case, in order to study the set $\Sigma \subset R$, we need the analogs of Proposition 2 and 3 about the admissible potentials. We will call $W=\left\{\lambda_{i}\right\}_{i \in \mathbb{Z}^{3}}$ an admissible potential if $\lambda_{i} \in \operatorname{supp} P_{q_{0}} \forall i \in \mathbb{Z}^{3}$ ( $P_{q_{0}}$ is the probability distribution of $q_{0}(\omega)$ ), and we will denote by $H_{\omega}$ the selfadjoint operator on $L^{2}\left(\mathbb{R}^{3}\right)$ whose resolvent is given by $(25)$ with $q_{k}(\omega)$ replaced by $\lambda_{k}$.

Proposition 5. Let $W$ be an admissible potential; then $\sigma\left(H_{w}\right) \subset \Sigma$.

The proof is omitted since it is identical to that of Proposition 2.

As before we will call $W=\left\{\lambda_{k}\right\}_{k \in \mathbb{Z}^{3}}$ a periodic admissible potential if there exists $L \in \mathbb{Z}^{3}$ such that $\lambda_{k+L}=\lambda_{k} \forall k \in \mathbb{Z}^{3}$, and we will denote by $P$ the class of the periodic admissible potentials. By repeating the proof of Proposition 3 we get:

\section{Proposition 6.}

$$
\Sigma=\overline{\bigcup_{w \in P} \sigma\left(H_{\omega}\right)} .
$$

The following two results about the periodic point interactions (see [9], Th 5.4, 5.1) will turn out to be useful: 
(i) Denote by $H_{\lambda}$ the periodic Hamiltonian corresponding to the resolvent (25) with $q_{k}(\omega)$ replaced by $\lambda \forall k \in \mathbb{Z}^{3}$.

If $\lambda<\lambda_{0}$ for some constant $\lambda_{0}$, then the spectrum of $H_{\lambda} \sigma\left(H_{\lambda}\right)$ is given by:

$$
\sigma\left(H_{\lambda}\right)=\left[E_{0}^{\lambda}, E_{1}^{\lambda}\right] \cup[0 \infty],
$$

with $E_{1}^{\lambda}<0$ and $E_{0}^{\lambda}, E_{1}^{\lambda}$ continuous monotone increasing functions of $\lambda$.

(ii) Let $A=\left\{n_{1} a_{1}+n_{2} a_{2}+n_{3} a_{3} ;\left(n_{1}, n_{2}, n_{3}\right) \mathbb{Z}^{3}\right\}$ where $a_{1}, a_{2}, a_{3}$ are three independent vectors in $\mathbb{Z}^{3}$; without loss of generality we can assume $a_{1}, a_{2}, a_{3}$ to be orthonormal, and let $X$ be a finite subset of $\mathbb{Z}^{3}$. Let $\lambda$ be a real function on $Y=A+X$ invariant under $A$, i.e. $\lambda_{\alpha+x}=\lambda_{x}, x \in X, \alpha \in A$. Then the Hamiltonian $H_{\lambda}$ whose resolvent is given by (31) with $q_{k}(\omega)$ replaced by $\lambda_{k} k \in \mathbb{Z}^{3}$ is invariant under translation in $A$ so that:

$$
H_{\lambda}=\frac{1}{|B|} \int_{B} H_{\lambda}(k) d k
$$

where $B=\left\{s_{1} b_{1}+s_{2} b_{2}+s_{3} b_{3},-1 / 2<s_{i} \leqq 1 / 2\right\},\left(b_{i}, a_{j}\right)=2 \pi \delta_{i j}$, is the Brillouin zone. The reduced Hamiltonians $H_{\lambda}(k)$ are selfadjoint operators on $l_{2}(\Gamma), \Gamma=\left\{n_{1} b_{1}\right.$ $\left.+n_{2} b_{2}+n_{3} b_{3},\left(n_{1}, n_{2}, n_{3}\right) \in \mathbb{Z}^{3}\right\}$ is the orthogonal lattice, whose resolvent is given by: $\left(H_{\lambda}(k)-E\right)^{-1}=\left((y+k)^{2}-E\right)^{-1}$

$$
-\sum_{x, y \in X}\left[\lambda_{x} \delta_{x y}-g_{E}(x-y, k)\right]^{-1} \frac{e^{i(\gamma+k) x-i\left(\gamma^{\prime}+k\right) y}}{\left((\gamma+k)^{2}-E\right)\left(\left(\gamma^{\prime}+k\right)^{2}-E\right)}(2 \pi)^{-3},
$$

$\gamma, \gamma^{\prime} \in \Gamma, k \in B$, where

$$
g_{E}(x-y, k)=(2)^{-3} \sum_{\gamma \in \Gamma} \frac{e^{i(\gamma+k)(x-y)}}{(\gamma+k)^{2}-E}
$$

if $x-y \neq 0$ and

$$
g_{E}(o, k)=\lim _{\omega \rightarrow \infty}(2 \pi)^{-3}\left[\sum_{\substack{\gamma \in \Gamma \\|\gamma+k|<\omega}} \frac{1}{|\gamma+k|^{2}-E}-4 \pi \omega\right]
$$

and []$^{-1}$ is the inverse of the $n \times n$ matrix

$$
\lambda_{x} \delta_{x y}-g_{E}(x-y, k), x, y \in X, n=|X| \text {. }
$$

Furthermore $H_{\lambda}(k)$ has discrete spectrum with eigenvalues $E_{n}(k, \lambda)$; the negative eigenvalues $E_{n}(k, \lambda)$ are the poles of $\left[\lambda_{x} \delta_{x y}-g_{E}(x-y, k)\right]^{-1}$. From this we get easily the following:

Proposition 7. Let the Hamiltonian $H_{\lambda}$ be as above (point (ii)) and suppose the periodic function $\lambda$ on $Y=A+X \subset \mathbb{Z}^{3}$ be such that : $-\infty<\lambda^{\min } \leqq \lambda_{x} \leqq \lambda^{\max } \leqq \lambda_{0} \forall x \in X$ (for the definition of $\lambda_{0}$, see point (i)). Then for any $\widetilde{E} \in \sigma\left(H_{\lambda}\right), \widetilde{E}<0$, there exists a function $\bar{\lambda}$ on $\mathbb{Z}^{3}, \bar{\lambda}_{j}=\bar{\lambda} \forall j \in \mathbb{Z}^{3}$ such that : $\widetilde{E} \in \sigma\left(H_{\lambda}\right)$.

Proof. By the previous result (point (ii)) we know that $\tilde{E}$ is a pole of $\left[\lambda_{x} \delta_{x y}-g_{E}\right.$ $(x-y, k)], x, y \in X$, i.e. there is one of the eigenvalues $\varepsilon_{m}^{\lambda}(\widetilde{E}, k)$ of the $n \times n$ matrix 
$\left[\lambda_{x} \delta_{x y}-g_{E}(x-y, k)\right]$ which is zero. Let us denote it by $\varepsilon_{m_{1}}^{\lambda}\left(\widetilde{E}, k_{1}\right)$. Since by the minimax-principle $\varepsilon_{m}^{\lambda}(\widetilde{E}, k)$ are monotone increasing functions of $\left\{\lambda_{x}\right\}_{x \in X}$, in the sense that if $\mu \geqq \lambda$ pointwise in $X$, then $\varepsilon_{n}^{\mu}(\widetilde{E}, k) \geqq \varepsilon_{n}(\widetilde{E}, k)$, we get:

$$
\varepsilon_{1}^{\lambda \min }\left(\widetilde{E}, k_{1}\right) \leqq \varepsilon_{m_{1}}^{\lambda}\left(\widetilde{E}, k_{1}\right) \leqq \varepsilon_{m_{1}}^{\lambda \max }\left(\tilde{E}, k_{1}\right),
$$

and thus, by the continuity, there exists a $\bar{\lambda}\left(m_{1}, k_{1}, \widetilde{E}\right)$ such that $\varepsilon_{m_{2}}^{\lambda}\left(\widetilde{E}, k_{1}\right)=0$, where $\varepsilon_{m}^{\lambda}(E, k)$ is the $m^{t h}$ eigenvalue of the matrix $\left[\lambda \delta_{x y}-g_{E}(x-y, k)\right]$. This means that $\widetilde{E} \in \sigma\left(H^{\lambda}\left(k_{2}\right)\right)$, i.e. $\tilde{E} \in \sigma\left(H^{\lambda}\right)$.

We are now in a position to prove the main result.

Proposition 8. Let the random variables $\left\{q_{i}(\omega)\right\}_{i \in \mathbb{Z}^{3}}$ be such that supp $P_{q_{0}} \subset\left[q_{\min }, q_{\max }\right]$ with $q_{\max }<\lambda_{0}$.

Then

$$
\left(E_{1}^{q_{\max }}, 0\right) \cap \Sigma=\varnothing,
$$

when $E_{1}^{q_{\max }}$ is the upper edge of the negative band in the spectrum of $H_{q_{\max }}$ Furthermore if $q_{\min }\left(q_{\max }\right)$ is the inf (sup) of supp $P_{q_{0}}$ and if

$$
\left[E_{0}^{q_{\min }}, E_{1}^{q_{\min }}\right] \cap\left[E_{0}^{q_{\max }}, E_{1}^{q_{\max }}\right] \neq \varnothing,
$$

then

$$
\Sigma=\sigma\left(H_{q_{\min }}\right) \cup \sigma\left(H_{q_{\max }}\right) .
$$

Proof. From Proposition 6 we know that $\Sigma=\overline{\bigcup_{W \in P} \sigma\left(H_{W}\right)}$, and from Proposition 7 we have that any $\widetilde{E} \in \sigma\left(H_{W}\right), \widetilde{E}<0, W \in P$ belongs to the spectrum of some $H_{\tilde{\lambda}(\tilde{E})}$; hence $E_{0}^{q_{\min }} \leqq \widetilde{E} \leqq E_{1}^{q_{\min }}$ since both $E_{0}^{\lambda}$ and $E_{1}^{\lambda}$ are monotone increasing functions of $\lambda$. From this it follows that

$$
\left(E_{1}^{q_{\max }}, 0\right) \cap \sigma\left(H_{W}\right)=\varnothing \quad \forall W \in P,
$$

and thus $\left(E_{1}^{q_{\max }}, 0\right) \cap \Sigma=\varnothing$.

If now $q^{\operatorname{mn}}\left(q^{\max }\right)$ is the $\inf (\sup )$ of $\operatorname{supp} P_{q_{0}}$, then $\sigma\left(H_{q_{\min }}\right) \subset \sum$ and the same for $\sigma\left(H_{q_{\max }}\right)$.

Since $\sigma\left(H_{q_{\min }}\right) \cup \sigma\left(H_{q_{\max }}\right)=\left[E_{0}^{q_{\min }}, E_{1}^{q_{\max }}\right] \cup[0, \infty[$, by the previous discussion we get that

$$
\sigma\left(H_{W}\right) \subset \sigma\left(H_{q_{\min }}\right) \cup \sigma\left(H_{q_{\max }}\right) \quad \forall W \in P,
$$

hence $\Sigma \subset \sigma\left(H_{q_{\min }}\right) \cup \sigma\left(H_{q_{\max }}\right)$ and by Proposition 5 we get

$$
\Sigma=\sigma\left(H_{q_{\min }}\right) \cup \sigma\left(H_{q_{\max }}\right) .
$$

Acknowledgements. It is a pleasure for us to thank Prof. S. Albeverio for his kind constant attention, numerous useful comments and helpful discussions. We also like to thank Prof. M. Fukushima and Prof. R. H $\phi$ egh-Krohn for instructive discussions and encouragements. This work has been done during the second named author's stay at the Fakultat für Physik at the University of Bielefeld and the Universitätsschwerpunkt Mathematisierung at the same university. The second named author is grateful to these institutions, and in particular to Profs. L. Streit and Ph. Blanchard for their warm hospitality and financial support. Finally we would like to thank Mrs. Siefert for the excellent typing of this paper. 


\section{References}

1. Albeverio, S., Fenstad J. E., Hфegh-Krohn, R.: Singular perturbations and nonstandard analysis. Tran. Am. Math. Soc. 252, 275-295 (1979)

2. Albeverio, S., Hфegh-Krohn, R.: Point interactions as Limits of short range interaction, Preprint, Bochum, 1980

3. Avron, J. E., Grossmann, A., Rodriguez, R.: Spectral properties of reduced Bloch Hamiltonians. Ann. Phys. 103, 47-63 (1977)

4. Borland, R. E. : Existence of energy gaps in one-dimensional liquids. Proc. Phys. Soc. (London) 78, 926 (1960)

5. Faris, W. G. : Self-adjoint operators. In: Lecture Notes in Mathematics Vol. 433, Berlin: Springer 1975

6. Flügge, S. : Rechenmethoden der Quantenmechanik, Berlin: Springer 1965

7. Frisch, H. L., Lloyd, S. P.: Electron levels in a one-dimensional random lattice. Phys. Rev. 120, 1175 1189 (1960); reprinted in [14]

8. Fakushima, M., Nagai, H., Nakao, S.: On an asymptotic property of spectra of random difference operator, Proc. Jpn. Acad. 51, 100-102 (1975)

9. Grossman, A., Hфegh-Krohn, R., Mebkhout, M.: The one particle theory of point interactions; Commun. Math. Phys. 77, 87-110 (1980)

10. Halperin, B. I. : Properties of a particle in a one-dimensional random potential. Adv. Chem. Phys. 13, 123-178 (1967)

11. Kirsch, W., Martinelli, F.: On the ergodic properties of the spectrum of general random operators. Preprint, Bochum, 1980

12. Kronig, R. de L., Penney, W. G.: Quantum mechanics of electrons in crystal lattices. Proc. R. Soc. (London) A130, 499-513 (1931); reprinted in [14]

13. Kunz, H.; Souillard, B.: Sur le spectre des opérateurs aux différences finies aléatoires, Commun. Math. Phys. 78, 201-246 (1980)

14. Lieb, E. H., Mattis, D. C. : Mathematical physics in one dimension. New York: Academic Press 1966

15. Luttinger, J. M.: Wave propagation in one-dimensional structure. Philips Res. Rep. 6, 303-310 (1951); reprinted in [14].

16. Morgan, J. D. III: Schrödinger operators whose potentials have separated singularities. J. Operat. Theory 1, 109-115 (1979)

17. Nakao, S.: On the spectral distribution of the Schrödinger operator with random potential. Jpn. J. Math. 3, 111-139 (1977)

18. Pastur, L. A.: Spectra of random selfadjoint operators. Russ. Math. Surv. 28, 1-67 (1973)

19. Pastur, L. A.: Spectral properties of disordered systems in the one-body approximation. Commun. Math. Phys. 75, 179-196 (1980)

20. Reed, M., Simon, B.: Methods of modern mathematical physics, Vol. I, II, IV. New York: Academic Press 1978

21. Saxon, D. S., Hutner, R. A. : Some electronic properties of a one-dimensional crystal model. Philips Res. Rep. 4, 81-122 (1949)

22. Weidmann, J. : Lineare Operatoren in Hilberträumen. Stuttgart: Teubner 1976 (english translation: Linear operators in Hilbert spaces Berlin: Springer 1980)

Communicated by Ya. G. Sinai

Received June 29, 1981; in revised form November 26, 1981 